\title{
Anthropometrical Measurements for Three-Dimensional Clothing Design
}

\author{
Inga DĀBOLIN̦A*, Ausma VIL̨UMSONE, Jānis DĀBOLIN̦Š \\ Institute of Textile Materials Technology and Design, \\ Riga Technical University, 14/24-319, Azenes str., Riga, LV 1048, Latvia
}

\begin{abstract}
Anthropometrical data can be acquired with different tools. Traditional methods use different manual tools (measuring tape, anthropometer, a.o.). as the technologies develop, new tools are created and/or the existent ones are improved. The scanning systems for human body measure acquisition uses different data acquisition methods. A human body surface reproduction dot cloud is created from the coordinate readings, which can be used as a virtual mannequin or only the coordinates themselves can be used. A virtual reproduction of the human body can be used in garment production, car production, engineering and medicine.

There is still not enough research and results as to use virtual mannequins for 3D garment designing. Mostly 3D scanning results are used to generate measures used in tailoring to use them in traditional or computer aided constructing methods. The surface of the human body is an object that cannot be laid out due to the vagueness of its surface and different variations. The human body consists of many different geometrical figures, it is a complicated shape and its laying out is a complicated process.

The designing of clothes includes a row of processes and one of the most time and labour consuming is constructing. A construction displays the layout of the surface of the body (garment). At present the existent garment construction ways do not provide a possibility of creating a garment without fitting, besides the plane-like projection process of a spatial object demands high level skill, imagination and creativity from the constructor.
\end{abstract}

An algorithm for the development of the geometric shape of a virtual mannequin has been worked out.

Keywords: 3D body scanning, spatial mannequin, clothing design, anthropometrical measurements

\section{Introduction}

3D scanning of the human body can be used successfully in computer aided garment designing and individualized production. The scanning of human body as a method for gaining human measurements has several preferences. The gathering of data is possible in a very short time. In comparison to manual measuring methods, scanning acquires a larger amount of measurements.

There are several modes of gaining human body measurements using the scanning system: laser scanning, light beam scanning, etc. The scanning systems for human body measure acquisition uses different data acquisition methods: dynamic range (lights and darks), laser beams, a.o.[1,2].

\section{Possibilities of acquiring anthropometrical data}

There are two types of human body measurement acquiring methods (Fig.1.):

1. manual anthropometry methods (contact methods);

2. optic anthropometry methods (non-contact methods).

Anthropometrical data can be acquired with different tools. Traditional methods use different manual tools (measuring tape, anthropometer, a.o.). as the technologies develop, new tools are created and/or the existent ones are improved. The electronic measuring tape can be considered as an improvement of the electronic measuring tape, but its use for anthropometry is doubtful. It has been proved by an experiment that when using the electronic measuring tape, more than half of the measures taken were wrong.

Photo measuring methods are fast and effective, but the processing of data is time consuming and labour intensive. A relatively new tool (approximately since 1980 [3]) in anthropometry is the 3D scanner.

Considering the advantages of 3D scanning, the scanning technologies are being developed and improved. Most of the scanners can not only create a 3D image of the human body, but also read the $\mathrm{x}$, $y$ and $z$ coordinates thereby acquiring precise information about the human body an its volumes [4].

\footnotetext{
* inga.dabolina@rtu.Iv+371 29364004
} 
A human body surface reproduction dot cloud is created from the coordinate readings, which can be used as a virtual mannequin or only the coordinates themselves can be used. A virtual reproduction of the human body can be used in garment production, car production, engineering and medicine.

Any scanning device is equipped with optic (light) appliances to ensure non-contact measuring. Such optic measurement acquisition devices can be divided into categories: photogrammetry, silhouette methods, laser scanning, light projection, electromagnetic wave and hybrid methods.

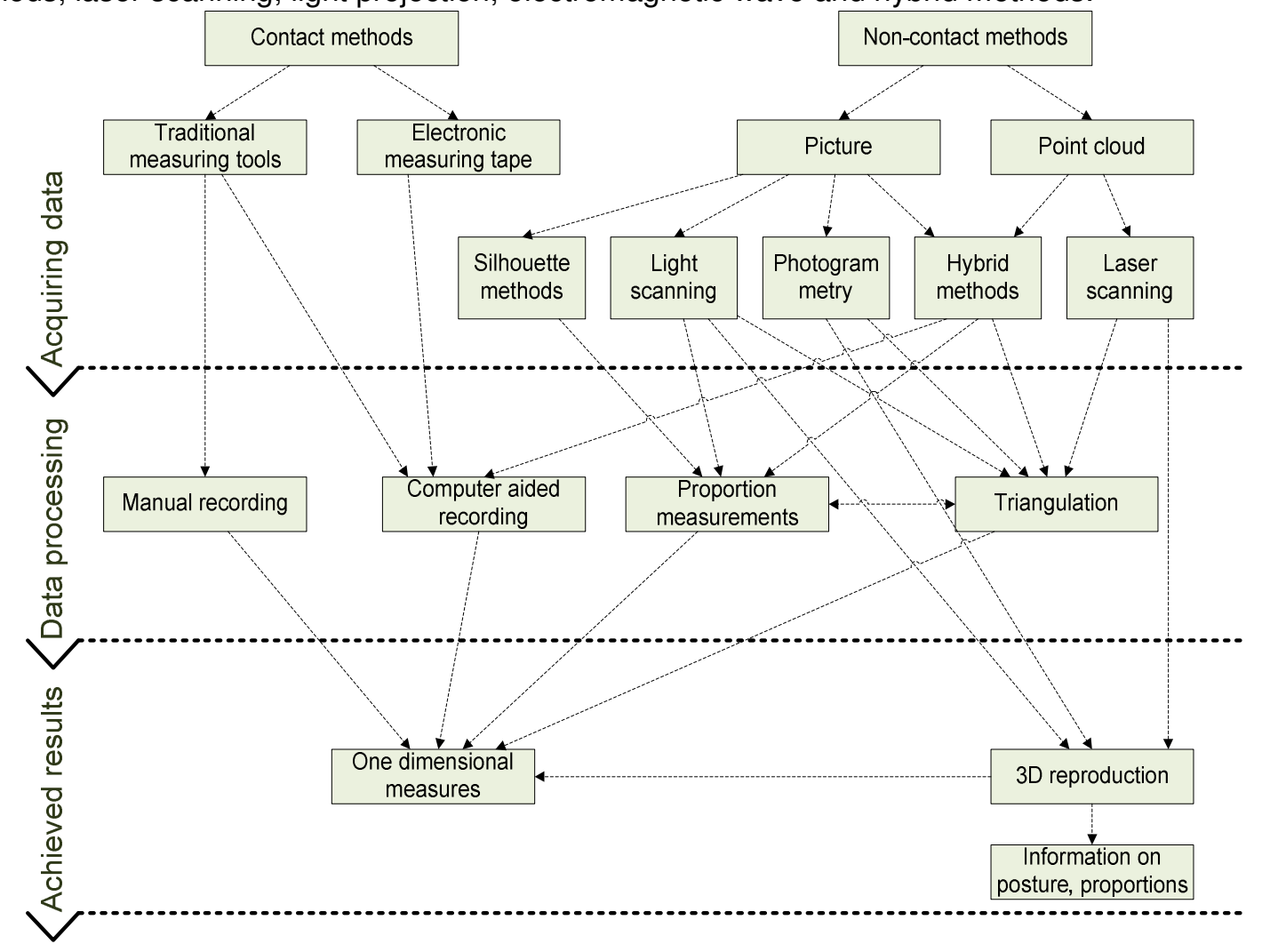

Fig. 1 Types of anthropometrical data acquisition

Each method has its advantages and disadvantages. In spite of the fact that laser scanning has been recognized as the most precise method and the gathered results are the most extensive (human body measurement data, a 3D virtual mannequin, a reflection of the actual texture, surface relief measurements, etc.), the light projection method is used more widely in the garment production industry since the equipment is much more cheaper than a laser scanner.

Comparing different anthropometrical data acquisition methods, the paper defines universal comparison criteria, qualitative and quantitative informative features: measures of the whole body at once, choice of the size of garment, creation of a 3D virtual mannequin, free (public) access, no postures, sizes and other limitations, data acquisition precision, data acquisition time, data processing time, computerization of data acquisition, no special equipment, easy to transport, easy to set up, low equipment cost. Such criteria and features allow performing a comparison of different anthropometrical data acquisition method functional characteristics. Following methods have been compared and analyzed: manual measurements, measurements with an e-measuring tape, photogrammetry, silhouette methods, laser scanning, light projection, electromagnetic wave method, and hybrid systems.

There is still not enough research and results as to use virtual mannequins for 3D garment designing. Mostly 3D scanning results are used to generate measures used in tailoring to use them in traditional or computer aided constructing methods.

\section{Human body surface layout possibilities}

The surface of the human body is an object that cannot be laid out due to the vagueness of its surface and different variations. The human body consists of many different geometrical figures, it is a complicated shape and its laying out is a complicated process.

The chapter describes the possibilities of laying out the surface of a mannequin or human body, dividing it into geometrical shapes which are possible to reproduce in a plane. 
With the development of 3D surface acquisition and lay out methods the triangle method is used most of the time. It is a plan base point web creation method, where the base points are placed in a way that they shape a mutually linked triangle system.

Base points are put on the irregular surface of the human body and then triangle plane points are being searched to be able to lay out the surface in a plane. This way it is possible to lay out a spatial surface in a plane.

To create a spread triangles are taken sequentially. Necessary data for each triangle is calculated (length of the edges, angles). Each peak of the triangle has the $x, y$ and $z$ coordinates. Wherewith it is possible to do calculations (see triangle example in figure 2).

$$
A B=\sqrt{\Delta x^{2}+\Delta y^{2}+\Delta z^{2}}
$$

where

$\Delta x=x_{A}-x_{E}$

Similarly it is possible to calculate the length of all edges $(A B, B C$, $A C)$, Knowing $A B, B C$ and $A C$ it is possible to calculate the $\operatorname{Cos} \alpha$ of the triangle for the angle shown in figure 2:

$\cos \alpha=\frac{A B^{2}+A C^{2}-B C^{2}}{2 A A B A C}$

Knowing the $\operatorname{Cos} \alpha$, the height KB of the triangle can be determined.

When performing the triangulation for the layout of s surface, the triangle arrangement principle is very important. The principle of extremes is used most of the time, where the peaks of the triangles are determined by the highest and lowest points in the nearest area, previously defining the maximum distance between the points. The higher precision wants to be achieved and the more spherical the surface, the smaller the lengths of the triangles have to be.

The geodesic method is also used to lay out the surface of the human body. The layout is created for the part of the surface marked by the geodesic lines. It is important to choose correct directions of the lines, the distance between them, as well as to consider the perpendicularity in the centre of the surface. Expressing it mathematically - geodesic lines are the generation of straight lines on a curved surface. Such lines are drawn with an inkling of that they are straight - namely two dimensional, they can be described with the help of the coordinates of the plane. A straight line drawn on a spherical surface, depending on the spherical level, creates one of the curved lines function (spline, hyperbole, etc.).

The plane splitting method uses the possibility of laying out geometrical planes. Each of the surface details isolated on the figure is equalled to a geometric surface which is laid out in a plane afterwards. The process consists of three stages: studying of the surface determining the splitting areas, determination and measuring of the splitting planes, drawing the spread.

The plane splitting method does not have a particular shape of the figures allowing free improvisation depending on the regularity of the surface or contrary - irregularity. That way this method as if combines the triangulation method and the geodesic line method.

Comparing the results of method usage and the workflow it can be concluded that none of the manually executable methods provides a precise result, the plane splitting and geodesic line method do not provide information about a rational positioning and size of seams, in its turn the triangulation has to be performed with small triangles so its usage and result would be appropriate. Besides as a result of the usage of the plane splitting and geodesic line methods surface describing plane parts with curved lines are acquired, not allowing to connect them properly. Nevertheless considering the possibilities of computerization it is being considered that the triangulation method describes the surface the best. Besides the triangle is the only plane shape that is unequivocal (it is only possible to draw one plane through three points without bending or breaking it). Therefore triangulation can be considered as the most precise engineering method for displaying a spatial surface in a plane.

\section{Development of a mannequin and definition of starting data for 3D garment designing}

The designing of clothes includes a row of processes and one of the most time and labour consuming is constructing. A construction displays the layout of the surface of the body (garment). At present the existent garment construction ways do not provide a possibility of creating a garment without fitting, besides the plane-like projection process of a spatial object demands high level skill, imagination and 
creativity from the constructor. Similarly the traditional human body measurement acquisition methods do not provide the acquisition of correct entry data.

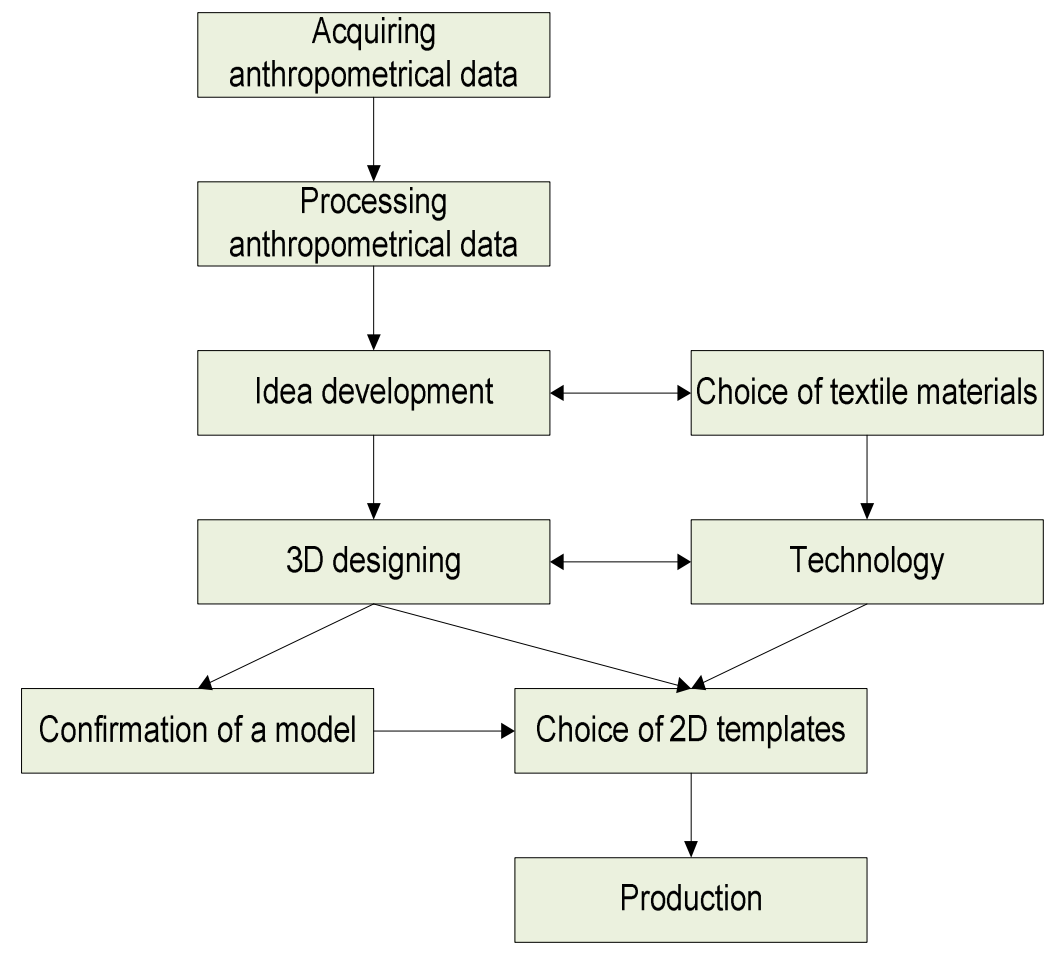

Fig. 3 The 3D process of garment designing

Therefore 3D designing is necessary with options like:

- Usage of a 3D virtual mannequin in designing - the optic human body measurement acquisition methods unbar the possibility to create a 3D mannequin automatically [5]. That will exclude the need for manual measurements.

- 3D spatial allowance designing, ensuring the comfort of the garment as well as the clarity of the visual design, excluding setting, which is a very labour intensive process with interactive repetitions.

- A visually graphic designing process - the possibility to evaluate the external looks of the garment would exclude the necessity of creating expensive patterns.

- The possibility to create details of the garment in a 3D environment following a layout in a plane.

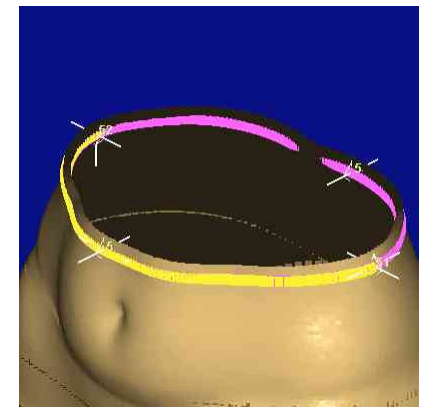

A

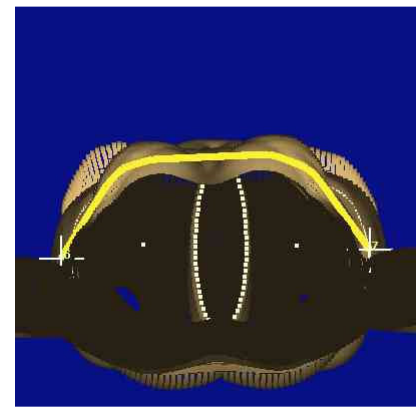

B

Fig.4 Acquiring anthropometrical measurements: a) waste girth, b) breast width

Such a designing process can be defined as a scheme of some actions:

To implement the technology that would allow designing clothes in a 3D environment, it is necessary to use an effective method for acquiring human body measurements, which would be appropriate for creating a digital model of the human body on a computer. 
3D parameters, information on the posture and proportions, as well as the measures used in tailoring are all acquired using a scanner. 1 The anthropometrical measures are acquired with the help of perimeter splits of a virtual mannequin, measurements of intermediate anthropometric point straight lines, as well as in the result of a combination and approximations of these versions (in case of breast width measurements a.o.) [6] (Figure 4). $3 \mathrm{D}$ data in three resolution levels are achieved after processing the data - the lowest resolution ( 26 000 dots), highest resolution $(\sim 70000$ dots) and a 3D mannequin with texture. Two types of parameters are acquired in each file:

- The $x, y$ and $z$ values of the 3D coordinates (marked with the letter v),

- The connection sequence of triangles (marked with the letter $f$ ).

The body surface point coordinates acquired by scanning are used to develop a 3D mannequin. The file with the coordinate parameters is saved and sub-files for point coordinate and triangle position determination are created. After this process the system cyclically reads the positions of points, the sequence and distances of triangles and places the points. The mannequins' surface is compiled from the placed dots and the individual 3D mannequin is created.

To create the surface of a garment it is necessary to determine the values of spatial allowances, which are defined as the distance between the garment and the surface of the body. To calculate these, traditionally used allowances can be used, or partially particular anthropometric point measurements in dynamics. The paper determines the coherence between the values of spatial allowances and traditionally used allowances. The paper also shows a possibility of using anthropometrically important point coordinate measurements of dynamic postures for the determination of ease allowances.

The 3D mannequin development system has been approbated by creating a virtual mannequin from the scanned data. A description of the creation of the geometric shape of a virtual mannequin has been developed. The spatial mannequin matches the actual body; the acquired measurements do not have significant deviations from the real ones. The developed 3D mannequin can be used in further research creating a 3D designing system.

\footnotetext{
1 The human body laser scanner VitusPro has been used to perform an experiment for 3D data acquisition. The research has been performed in cooperation with the scientific research laboratory of the University of Switzerland MiraLab. Switzerland, Geneva. 18.05. - 21.05.2008.
} 


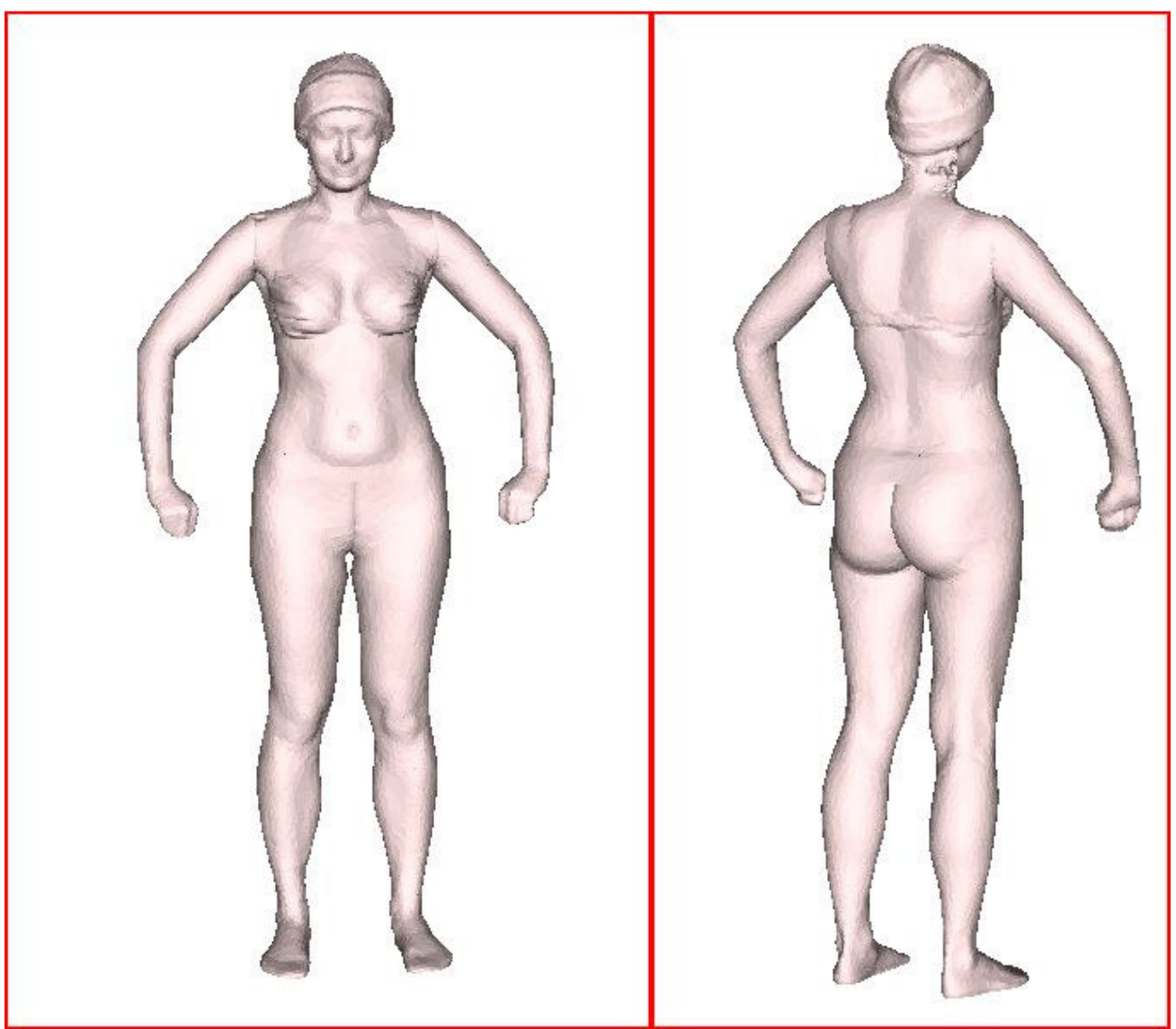

Fig.6. The created 3D mannequin

\section{Conclusions}

As a result of the analysis of the existent clothing designing process, its methods and techniques it has been concluded that a 3D garment designing system has to be equipped with a 3D mannequin, a Information on 3D human body data acquisition possibilities has been systemized.

spatial allowance system and clothing surface definition means.

Computer aided clothing designing and anthropometrical data acquisition possibilities available for use, systems and methods have been studied and analyzed in the paper.

The possibilities of 3D human body scanning have been studied, identified and systemized, characteristics have been given and an analysis of possible combining has been performed. Methods of a 3D surface lay out have been studies, analyzed and compared.

The paper studies different possibilities of human surface lay out, and mannequin surface lay out engineering methods have been tested. The layout of a bodies' surface is necessary to determine the parameters of an object (in aspect of this paper - clothing). Since it is not possible to define particular individual geometrical figures of which the human body, and wherewith the clothing surface, consists it has been concluded that triangulation can be considered as the most precise engineering method for the reproduction of a surface in a plane.

The starting data of 3D designing have been systemized: spatial virtual mannequin, spatial allowances (comfort and modern), clothing coordinates; and their determination procedure have been defined. Examples for the recalculation of traditionally used allowances have been given.

An algorithm for the development of the geometric shape of a virtual mannequin has been worked out. The spatial mannequin matches the actual body, the measures do not have essential deviations from the actual ones. The created $3 \mathrm{D}$ mannequin allows developing a $3 \mathrm{D}$ designing system in further research. 


\section{Acknowledgements}

This work has been partly supported by the European Social Fund co-financed project "Establishment of interdisciplinary research groups for a new functional properties of smart textiles development and integrating in innovative products"

\section{References}

1. D'Apuzzo Nicola Feasibility study: Full Body Scanning, Virtual-Try-On, Face Scanning, Virtual-Make-Over with application in apparel. - Zürich, Switzerland : Hometrica Consulting, 2008.

2. Kang Tae Jin un Kim Sung Min Optimized garment pattern generation based on three-dimensional anthropometric measurement // International Journal of Clothing Science and Technology. 0955-6222 : MCB University Press Ltd, 2000. - 4. : Vol. 12.. - p. 240.-254.. - ISSN: 0955-6222.

3. Fan J. Yu W., Hunter L. Clothing appearance and fit: Science and technology [Book]. - Cambridge, England: Woodhead Publishing Limited, 2004. - p. 240. - ISBN 0-8493-2594-3.

4. Hwang Su-Jeong Three dimensional body scanning systems with potential for use in the apparel industry [Article]. - Raleigh, 2001. - p. 63.

5. Siegmund Jana [a.o.] Development of parametric virtual dummies; 3D patterning of corsetry [Magazine] // Textile Network. - Dresden: apparel, 2007, 7 - 8. - TU Dresden.

6. Human Solutions GmbH [Live]. - 3D human body measurement software. www.human-solutions.com. - Germany. 